\title{
An exploration of the antioxidant effects of garlic saponins in mouse-derived C2C12 myoblasts
}

\author{
JI SOOK KANG ${ }^{1}$, SUNG OK KIM ${ }^{2}$, GI-YOUNG KIM ${ }^{3}$, HYE JIN HWANG ${ }^{1,4}$, BYUNG WOO KIM ${ }^{1,5}$, \\ YOUNG-CHAE CHANG ${ }^{6}$, WUN-JAE KIM ${ }^{7}, \mathrm{CHEOL} \mathrm{MIN} \mathrm{KIM}^{8}$, YOUNG HYUN YOO ${ }^{9}$ and YUNG HYUN CHOI ${ }^{1,10}$ \\ ${ }^{1}$ Blue-Bio Industry RIC and Anti-Aging Research Center, Dongeui University, Busan 614-714; \\ ${ }^{2}$ Department of Food Science and Biotechnology, College of Engineering, Kyungsung University, Busan 608-736; \\ ${ }^{3}$ Laboratory of Immunobiology, Department of Marine Life Sciences, Jeju National University, Jeju 690-756; \\ ${ }^{4}$ Department of Food and Nutrition, College of Natural Sciences and Human Ecology, Dongeui University; \\ ${ }^{5}$ Department of Life Science and Biotechnology, College of Natural Sciences and Human Ecology, Dongeui University, \\ Busan 614-714; ${ }^{6}$ Research Institute of Biomedical Engineering and Department of Medicine, Catholic University \\ of Daegu School of Medicine, Daegu 705-718; ${ }^{7}$ Department of Urology, Chungbuk National University College of Medicine, \\ Cheongju 361-763; ${ }^{8}$ Department of Biochemistry, Busan National University College of Medicine, \\ Yangsan 626-870; ${ }^{9}$ Department of Anatomy and Cell Biology, Dong-A University College of Medicine \\ and Mitochondria Hub Regulation Center, Busan 602-714; ${ }^{10}$ Department of Biochemistry, \\ Dongeui University College of Korean Medicine, Busan 614-052, Republic of Korea
}

Received June 16, 2015; Accepted October 19, 2015

DOI: $10.3892 /$ ijmm.2015.2398

\begin{abstract}
In this study, we aimed to confirm the protective effects of garlic saponins against oxidative stress-induced cellular damage and to further elucidate the underlying mechanisms in mouse-derived $\mathrm{C} 2 \mathrm{C} 12$ myoblasts. Relative cell viability was determined by 3-(4.5-dimethylthiazol-2-yl)-2.5 diphenyltetrazolium bromide assay. Comet assay was used to measure DNA damage and oxidative stress was determined using 2',7'-dichlorofluorescein diacetate to measure intracellular reactive oxygen species (ROS) generation. Western blot analysis and small interfering RNA (siRNA)-based knockdown were used in order to investigate the possible molecular mechanisms. Our results revealed that garlic saponins prevented hydrogen peroxide $\left(\mathrm{H}_{2} \mathrm{O}_{2}\right)$-induced growth inhibition and exhibited scavenging activity against intracellular ROS. We also observed that garlic saponins prevented $\mathrm{H}_{2} \mathrm{O}_{2}$-induced comet tail formation and decreased the phosphorylation levels
\end{abstract}

Correspondence to: Dr Young Hyun Yoo, Department of Anatomy and Cell Biology, Dong-A University College of Medicine and Mitochondria Hub Regulation Center, 3-1 Dongdaesin-Dong, Seo-Gu, Busan 602-714, Republic of Korea

E-mail: yhyoo@dau.ac.kr

Dr Yung Hyun Choi, Department of Biochemistry, Dongeui University College of Korean Medicine, 42San, Yangjung-dong, Busan 614-052, Republic of Korea

E-mail: choiyh@deu.ac.kr

Key words: garlic saponins, oxidative stress, nuclear factor erythroid 2-related factor 2/heme oxygenase-1, extracellular signalregulated kinase of $\gamma \mathrm{H} 2 \mathrm{AX}$ expression, suggesting that they can prevent $\mathrm{H}_{2} \mathrm{O}_{2}$ induced DNA damage. In addition, garlic saponins increased the levels of heme oxygenase-1 (HO-1), a potent antioxidant enzyme associated with the induction and phosphorylation of nuclear factor erythroid 2-related factor 2 (Nrf2) and the translocation of Nrf2 from the cytosol into the nucleus. However, the protective effects of garlic saponins on $\mathrm{H}_{2} \mathrm{O}_{2}$-induced ROS generation and growth inhibition were significantly reduced by zinc protoporphyrin IX, an HO-1 competitive inhibitor. In addition, the potential of garlic saponins to mediate $\mathrm{HO}-1$ induction and protect against $\mathrm{H}_{2} \mathrm{O}_{2}$-mediated growth inhibition was adversely affected by transient transfection with Nrf2-specific siRNA. Garlic saponins activated extracellular signal-regulated kinase (ERK) signaling, whereas a specific ERK inhibitor was able to inhibit HO-1 upregulation, as well as Nrf2 induction and phosphorylation. Taken together, the findings of our study suggest that garlic saponins activate the Nrf2/HO-1 pathway by enabling ERK to contribute to the induction of phase II antioxidant and detoxifying enzymes, including HO-1 in C2C12 cells.

\section{Introduction}

Oxidative stress resulting from an imbalance between systemgenerating and scavenging reactive oxygen species (ROS) is the pathological basis of a number of chronic diseases. Low levels of ROS are scavenged effectively by the antioxidant defense system of cells. However, under conditions of oxidative stress, the excessive accumulation of ROS causes destructive and irreversible damage to cellular components, including nucleic acids, proteins and lipids, as well as to other macromolecules, which ultimately results in cell death $(1,2)$. As a result, the induction of antioxidant enzymes is one of the most important determinants of cytoprotective effects against oxidative stress. 
Nuclear factor erythroid 2-related factor 2 (Nrf2), a regulator of the antioxidant response, plays a critical role in protecting cells against oxidative stress. Under basal conditions, Nrf2 is sequestered and inactivated in the cytoplasm by binding to its inhibitor protein, Kelch-like ECH-associated protein 1 (Keap1), which functions as an adaptor for Cullin 3 (Cul3)-based E3 ligase in order to regulate the proteasomal degradation of Nrf2 $(3,4)$. When the complex is disrupted by exposure to various stimuli, free Nrf2 subsequently translocates into the nucleus, where it sequentially binds to the antioxidant response element (ARE) $(5,6)$. This results in a cytoprotective response, which is characterized by the induction of the gene expression of phase II enzymes. This response involves the induction of heme oxygenase-1 (HO-1) and $\mathrm{NAD}(\mathrm{P}) \mathrm{H}$ :quinone oxidoreductase 1 (NQO1), as well as decreased sensitivity to oxidative stress-induced damage $(3,7)$. Recent studies have indicated that the Nrf2 protein may be phosphorylated by several signal transduction pathways, including mitogen-activated protein kinases (MAPKs), phosphatidylinositol 3-kinase (PI3K)/Akt and protein kinase $\mathrm{C}(8-10)$. In this way, Nrf2 dissociates from Keap1 and translocates to the nucleus, where it activates the ARE region of promoters for numerous cytoprotective genes.

Certain toxic substances that are harmful to the human body are contained in raw materials used for food, and in order to discover new functional substances in the raw materials of food that humankind has long ingested, previous research has concentrated on such substances (11). In particular, for the prevention and treatment of diverse diseases, including, but not limited to, metabolic disorders, cancer, cardiovascular disease and Alzheimer's disease, caused by oxidative stress, rather than using artificially synthesized compounds, food derived from natural products can be a more useful potential therapy.

Garlic (Allium sativum L., Alliaceae) has been used as a food additive and herbal medicine for over 5,000 years, and is one of the earliest-documented herbs to be used for the maintenance of health and the treatment of disease. Previous studies have examined the close association between garlic intake and the occurrence of disease $(12,13)$. Garlic is known for its production of organosulphur compounds, as well as steroid saponins. Although organosulphur compounds, which are the major antioxidant components of garlic extract, have scavenging free radical properties and reduce lipid peroxidation, they are unstable and give rise to transformed products $(14,15)$. However, garlic saponins are more stable and thus are more suitable for cooking and storage, and have been found to be involved in various pharmacological activities (16-20). Previous studies have proven that garlic saponins are a potent antioxidant, protecting cells by reducing ROS production in response to oxidative stress $(18,19,21)$. For example, Luo et al (22) confirmed that garlic saponins functions as antioxidants to protect rat pheochromocytoma $\mathrm{PC} 12$ cells from the direct damage of hypoxia-induced ROS and exert protective effects through redox-sensitive signaling pathways mediated by ROS. These studies also hypothesized that Nrf2/ARE activation may be an important pathway for the activation of the catalase that is induced following treatment with garlic saponins. However, to the best of our knowledge, no study to date has suggested that garlic saponins may act both as an antioxidant for the direct elimination of ROS and as a signaling molecule for the activation of Nrf2/ARE. As a result, in this study, we aimed to investigate the antioxidant effects of garlic saponins.

The aim of the present study was to further examine the intracellular pathways involved in order to determine whether garlic saponins are able to activate $\mathrm{Nrf} 2$ and induce the expression of its downstream target genes in mouse-derived $\mathrm{C} 2 \mathrm{C} 12$ myoblasts stimulated with hydrogen peroxide $\left(\mathrm{H}_{2} \mathrm{O}_{2}\right)$.

\section{Materials and methods}

Cell culture and treatment with garlic saponins. C2C12 myoblasts obtained from the American Type Culture Collection (ATCC; Manassas, VA, USA) were grown in Dulbecco's modified Eagle's medium (DMEM; WelGENE Inc., Daegu, Korea), supplemented with $10 \%$ fetal bovine serum (FBS) and $100 \mu \mathrm{g} / \mathrm{ml}$ penicillin/streptomycin antibiotics (WelGENE Inc.) in a humidified $5 \% \mathrm{CO}_{2}$ atmosphere at $37^{\circ} \mathrm{C}$. For the preparation of the crude garlic saponins, an improved method was used for saponin extraction based on a previous study (22). Garlic was collected around Namhae city (Gyeongsangnam-do, Korea); the bulbs were peeled, washed and chopped before being stored at $-20^{\circ} \mathrm{C}$. The frozen samples were lyophilized and homogenized using a grinder before extraction. The samples were extracted twice with methanol by refluxing at $80^{\circ} \mathrm{C}$ for $2 \mathrm{~h}$. The methanol extract was then suspended in water and partitioned sequentially with $n$-hexane, chloroform, ethyl acetate and $n$-butanol. Subsequently, the water-saturated $n$-butanol fraction was evaporated to dryness in a vacuum. The crude saponins recovered in this process were loaded onto a Diaion ${ }^{\circledR}$ HP-20 MCI gel (SigmaAldrich Chemical Co., St. Louis, MO, USA). The sugar residues were then removed with $40 \% \mathrm{CH}_{3} \mathrm{OH}$. The fractions were eluted with $60-80 \% \mathrm{CH}_{3} \mathrm{OH}$, collected, and then dried to obtain the garlic saponins. The saponins were then dissolved in dimethyl sulfoxide (DMSO, Sigma-Aldrich Chemical Co.) and adjusted to final concentrations using complete DMEM prior to use.

Cell viability assay. Cell viability was measured based on the formation of blue formazan, which was metabolized from colorless 3-(4.5-dimethylthiazol-2-yl)-2.5 diphenyl-tetrazolium bromide (MTT; Sigma-Aldrich Chemical Co.) by mitochondrial dehydrogenases. These are active only in live cells. Briefly, the $\mathrm{C} 2 \mathrm{C} 12$ cells were seeded in 6-well plates at a density of $1 \times 10^{5}$ cells per well. After $24 \mathrm{~h}$ of incubation, the cells were treated with the specified concentrations of garlic saponins in the absence or presence of $\mathrm{H}_{2} \mathrm{O}_{2}$ and/or zinc protoporphyrin IX (ZnPP; Sigma-Aldrich Chemical Co.) and N-acetyl-L-cysteine (NAC; Sigma-Aldrich Chemical Co.) for the specified duration. MTT working solution was then added to the culture plates following by continuous incubation at $37^{\circ} \mathrm{C}$. Three hours later, the supernatant was removed, and the formation of formazan was measured at $540 \mathrm{~nm}$ using an enzyme-linked immunosorbent assay (ELISA) plate reader (Dynatech Laboratories, Chantilly, VA, USA). Control cells were supplemented with complete medium containing $0.05 \%$ DMSO (vehicle control). The inhibitory effect on cell growth was assessed as the percentage of cell viability, where the vehicle-treated cells were considered $100 \%$ viable.

Measurement of ROS production. The intracellular accumulation of ROS was determined using the fluorescent probes, 
2',7'-dichlorodihydrofluorescein diacetate $\left(\mathrm{H}_{2} \mathrm{DCFDA}\right.$; Molecular Probes, Eugene, OR, USA). In order to monitor ROS generation, the cells were incubated with $10 \mu \mathrm{M} \mathrm{H}_{2}$ DCFDA for $20 \mathrm{~min}$ at room temperature in the dark. ROS production in the cells was monitored using a flow cytometer (Becton Dickinson, San Jose, CA, USA) using CellQuest Pro software, as previously described (23).

Comet assay (single-cell gel electrophoresis). Comet assay, a sensitive and rapid technique for detection of DNA damage in individual cells, was performed as previously described (24). Briefly, harvested individual cells were mixed with molten low melt agarose and spread on a fully-frosted microscopic slide precoated with $1 \%$ normal melting agarose. The embedded cells were then lysed using lysis solution and treated with alkaline solution to relax and denature the DNA. Subsequently, electrophoresis of the samples was carried out under alkaline condition at $25 \mathrm{~V}$ and $300 \mathrm{~mA}$ for $20 \mathrm{~min}$. Following electrophoresis, the slides were washed, stained with $20 \mu \mathrm{g} / \mathrm{ml}$ propidium iodide (PI; Sigma-Aldrich Chemical Co.), and were then examined under a fluorescence microscope (Carl Zeiss, Jena, Germany).

Protein extraction, electrophoresis and western blot analysis. Western blot analysis and protein extraction were performed as previously described (24). In brief, the cells were lysed, and then equal amounts of cell lysates were separated on sodium dodecyl sulfate (SDS)-polyacrylamide gels and transferred onto nitrocellulose membranes (Schleicher \& Schuell Bioscience, Inc., Keene, NH, USA). The membranes were probed with specific antibodies for $1 \mathrm{~h}$ and incubated with the diluted enzyme-linked secondary antibodies (Amersham Co., Arlington Heights, IL, USA). The proteins were visualized using an enhanced chemiluminescence (ECL) detection system (Amersham Co.) according to the manufacturer's instructions. The primary antibodies used in this study were as follows: $\gamma \mathrm{H} 2 \mathrm{AX}$ (1:500, CS \#2577; rabbit polyclonal, Cell Signaling Technology, Inc., Danvers MA, USA), p- $\gamma \mathrm{H} 2 \mathrm{AX}$ (1:500, CS \#9718S; rabbit polyclonal, Cell Signaling Technology, Inc.), Nrf2 (1:500, SC-13032; rabbit polyclonal, Santa Cruz Biotechnology, Inc., Santa Cruz, CA, USA), p-Nrf2 (1:500, ab76026; rabbit monoclonal, Abcam, Inc., Cambridge, UK), HO-1 (1:500, SC-136960; mouse monoclonal, Santa Cruz Biotechnology, Inc.), Keap1 (1:1,000, SC-33569; rabbit polyclonal, Santa Cruz Biotechnology, Inc.), NQO-1 (1:1,000, SC-16464; goat polyclonal, Santa Cruz Biotechnology, Inc.), TrxR1 (1:1,000, SC-28321; mouse monoclonal, Santa Cruz Biotechnology, Inc.), ERK (1:1,000, SC-154; rabbit polyclonal, Santa Cruz Biotechnology, Inc.), p-ERK (1:500, \#9106S; mouse monoclonal, Cell Signaling Technology, Inc.), p38 (1:1,000, SC-535; rabbit polyclonal, Santa Cruz Biotechnology, Inc.), p-p38 (1:500, \#9211S; rabbit polyclonal, Cell Signaling Technology, Inc.), JNK (1:1,000, \#9252S; rabbit polyclonal, Cell Signaling Technology, Inc.), p-JNK (1:500, \#9255S; mouse monoclonal, Cell Signaling Technology, Inc.) and actin (1:1,000, SC-1616; goat polyclonal, Santa Cruz Biotechnology, Inc.). Actin and lamin B were used as the internal controls for cytosolic and nuclear fractions, respectively. In order to examine the effects of MAPK signaling pathway on the activation of Nrf2 and the induction of HO-1 by garlic saponins, specific inhibitors of MAPKs such as PD98059 (an ERK inhibitor,

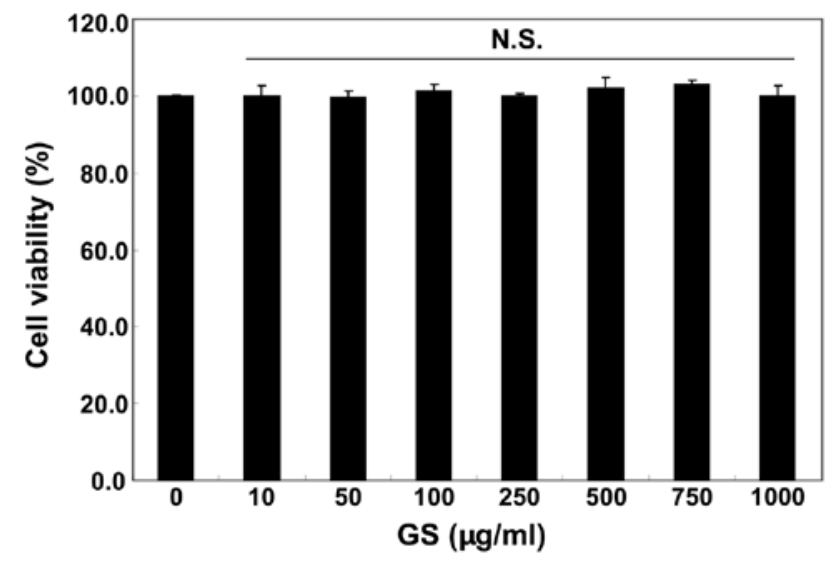

Figure 1. Effects of garlic saponins on the growth of $\mathrm{C} 2 \mathrm{C} 12$ cells. Cells were treated with various concentrations of garlic saponins for $24 \mathrm{~h}$. Cell viability was then assessed using an MTT reduction assay. The results are represented as the means \pm SD values obtained from 3 independent experiments. GS, garlic saponins.

Cell Signaling Technology, Inc.), SP600125 (a JNK inhibitor, Sigma-Aldrich Chemical Co.) and SB203580 (a p38 MAPK inhibitor, Cell Signaling Technology, Inc.) were applied.

Small interfering RNA (siRNA) transfection. siRNA targeting Nrf2 (Nrf2 siRNA) and control siRNA were purchased from Santa Cruz Biotechnology. The siRNA was transfected into the cells following the manufacturer's instructions using Lipofectamine 2000 Transfection Reagent (Life Technologies, Carlsbad, CA, USA). For transfection, the cells were seeded in 6-well culture plates and incubated with control siRNA or Nrf2 siRNA at $50 \mathrm{nM}$ for $6 \mathrm{~h}$ in serum-free OPTI-MEM medium (Life Technologies). Following transfection, the cells were treated with garlic saponins $(500 \mu \mathrm{g} / \mathrm{ml})$ for $6 \mathrm{~h}$ or pre-treated with garlic saponins $(500 \mu \mathrm{g} / \mathrm{ml})$ for $1 \mathrm{~h}$ and then stimulated with or without $1 \mathrm{mM} \mathrm{H}_{2} \mathrm{O}_{2}(1 \mathrm{mM})$ in the presence of garlic saponins for a further $6 \mathrm{~h}$. The cells were then lysed and equal amounts of cell lysates were subjected to western blot analysis.

Statistical analysis. Data are expressed as the means \pm standard deviation (SD) values. One-way analysis of variance (ANOVA) was used for comparisons in the experiments with multiple time points and concentrations. When ANOVA indicated statistical significance, Duncan's multiple range test was used to determine which means were significantly different. A probability value of $\mathrm{P}<0.05$ was used as the criterion for statistical significance.

\section{Results}

Garlic saponins protect $\mathrm{C} 2 \mathrm{C} 12$ cells from $\mathrm{H}_{2} \mathrm{O}_{2}$-induced cytotoxicity. We first examined the effects of garlic saponins on the viability of $\mathrm{C} 2 \mathrm{C} 12$ cells by MTT assay. As shown in Fig. 1, the results revealed that treatment with garlic saponins $(10-1,000 \mu \mathrm{g} / \mathrm{ml})$ alone had no obvious effect on $\mathrm{C} 2 \mathrm{C} 12$ cell viability. To examine the protective effects of garlic saponins against oxidative stress-induced cytotoxicity in $\mathrm{C} 2 \mathrm{C} 12$ cells, the cells were pre-treated with garlic saponins for $1 \mathrm{~h}$ and exposed to $\mathrm{H}_{2} \mathrm{O}_{2}$ for an additional $6 \mathrm{~h}$. The results revealed that treatment of the $\mathrm{C} 2 \mathrm{C} 12$ cells with $1 \mathrm{mM}^{\circ} \mathrm{H}_{2} \mathrm{O}_{2}$ for $6 \mathrm{~h}$ resulted 


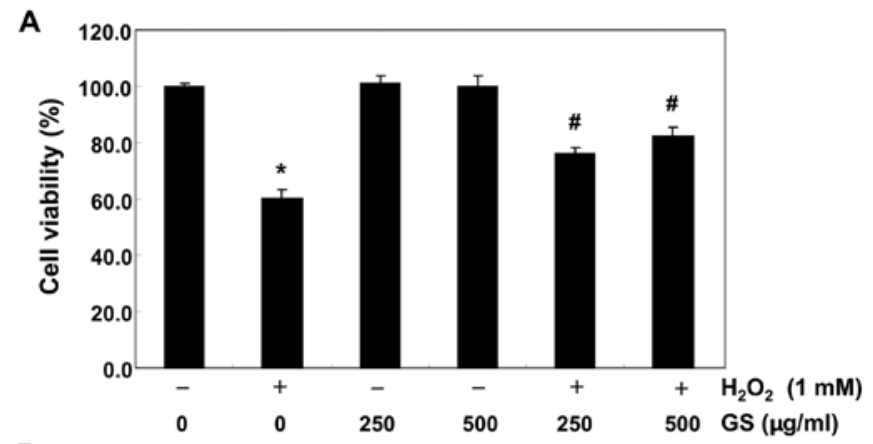

B

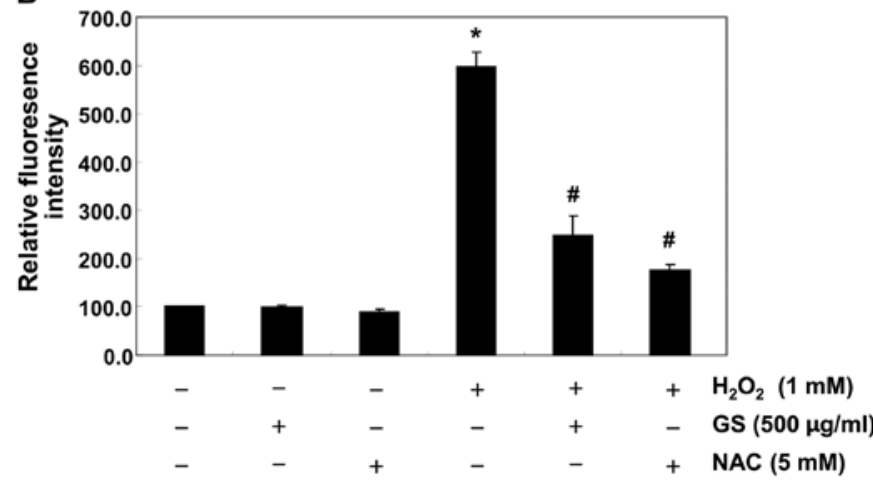

Figure 2. Garlic saponins attenuate $\mathrm{H}_{2} \mathrm{O}_{2}$-induced growth inhibition and reactive oxygen species (ROS) generation in $\mathrm{C} 2 \mathrm{C} 12$ cells. $\mathrm{C} 2 \mathrm{C} 12$ cells were pre-treated with the specified concentrations of garlic saponins or $5 \mathrm{mM} \mathrm{NAC}$ for $1 \mathrm{~h}$. The cells were then stimulated with or without $1 \mathrm{mM} \mathrm{H}_{2} \mathrm{O}_{2}$ in the presence of garlic saponins for a further $6 \mathrm{~h}$. (A) Cell viability was assessed using an MTT reduction assay. (B) In order to monitor ROS production, the cells were incubated at $37^{\circ} \mathrm{C}$ in the dark for 20 min with new culture medium containing $10 \mu \mathrm{M} \mathrm{H}_{2}$ DCFDA. ROS generation was measured using a flow cytometer. The results are presented as the means \pm SD values obtained from 3 independent experiments ( $\mathrm{P}<0.05$ compared with the control group; ${ }^{\prime \prime} \mathrm{P}<0.05$ compared with the $\mathrm{H}_{2} \mathrm{O}_{2}$-stimulated group). GS, garlic saponins.

in approximately a $40 \%$ loss of cellular viability, as compared with the control cells. However, the $\mathrm{H}_{2} \mathrm{O}_{2}$-induced reduction in cell viability was significantly reversed by pre-treatment with garlic saponins in a concentration-dependent manner (Fig. 2A). These results indicate that garlic saponins have properties that protect $\mathrm{C} 2 \mathrm{C} 12$ cells against oxidative stress.

Garlic saponins modulate $\mathrm{H}_{2} \mathrm{O}_{2}$-induced $\mathrm{ROS}$ generation in C2C12 cells. We then measured the intracellular ROS levels in order to investigate whether garlic saponins had any effect on intracellular ROS generation induced by stimulation with $\mathrm{H}_{2} \mathrm{O}_{2}$. As expected, exposure of the $\mathrm{C} 2 \mathrm{C} 12$ cells to $\mathrm{H}_{2} \mathrm{O}_{2}$ for $6 \mathrm{~h}$ induced an increase in intracellular ROS levels (Fig. 2B). However, pretreatment of the cells with garlic saponins $(500 \mu \mathrm{g} / \mathrm{ml}$ for $1 \mathrm{~h})$ significantly reduced the $\mathrm{H}_{2} \mathrm{O}_{2}$-induced ROS production. As a positive control, the ROS scavenger, NAC, was used, and we noted that this also reduced $\mathrm{H}_{2} \mathrm{O}_{2}$-induced ROS generation. Moreover, we noted that the garlic saponins themselves did not contribute to ROS generation, suggesting that pre-treatment with garlic saponins induced a cellular antioxidant response.

Garlic saponins attenuate $\mathrm{H}_{2} \mathrm{O}_{2}$-induced DNA damage in C2C12 cells. We further examined the effects of garlic saponins on DNA damage induced by $\mathrm{H}_{2} \mathrm{O}_{2}$ using single-cell gel electrophoresis (comet assay) and western blot analysis. As
A
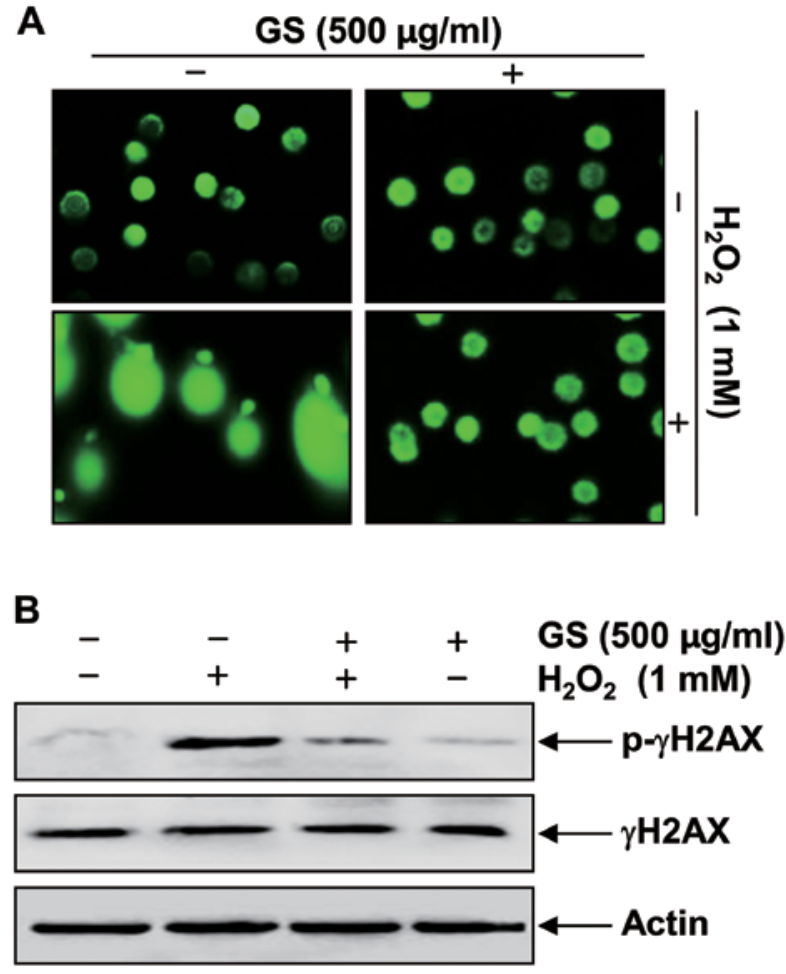

Figure 3. Garlic saponins protect against $\mathrm{H}_{2} \mathrm{O}_{2}$-induced DNA damage in $\mathrm{C} 2 \mathrm{C} 12$ cells. C2C12 cells were pre-treated with a $500 \mu \mathrm{g} / \mathrm{ml}$ of garlic saponins for $1 \mathrm{~h}$. The cells were then stimulated with or without $1 \mathrm{mM} \mathrm{H}_{2} \mathrm{O}_{2}$ in the presence of garlic saponins for a further $6 \mathrm{~h}$. (A) To detect cellular DNA damage, comet assay was performed and representative images of the comets were taken using a fluorescence microscope (x200, original magnification). (B) The cells were lysed and then equal amounts of cell lysates were separated on SDS-polyacrylamide gels and transferred to nitrocellulose membranes. The membranes were probed with specific antibodies against $\mathrm{p}-\gamma \mathrm{H} 2 \mathrm{AX}, \gamma \mathrm{H} 2 \mathrm{AX}$ and actin, as an internal control. The proteins were visualized using an ECL detection system. A representative blot from 3 independent experiments is shown. GS, garlic saponins.

shown in Fig. 3A, stimulation with $\mathrm{H}_{2} \mathrm{O}_{2}$ alone significantly increased the number of DNA breaks, resulting in an increase in fluorescence intensity in the tails of the comet-like structures in $\mathrm{C} 2 \mathrm{C} 12$ cells. These adverse effects were markedly reduced by pre-treatment with garlic saponins. In addition, stimulation of the $\mathrm{C} 2 \mathrm{C} 12$ cells with $\mathrm{H}_{2} \mathrm{O}_{2}$ alone resulted in the upregulation of the level of the phosphorylated histone variant $\mathrm{H} 2 \mathrm{AX}$ at serine 139 ( $\mathrm{p}-\gamma \mathrm{H} 2 \mathrm{AX})$, a sensitive marker for DNA doublestrand breaks (25) (Fig. 3B). By contrast, pre-treatment with garlic saponins resulted in a decreased $\mathrm{p}-\gamma \mathrm{H} 2 \mathrm{AX}$ expression, which again indicates that garlic saponins exert a protective effect against $\mathrm{H}_{2} \mathrm{O}_{2}$-induced DNA damage.

Garlic saponins enhance the expression of Nrf2 and HO-1 in C2C12 cells. The fact that Nrf2 signaling regulates the cellular antioxidant response by promoting ARE-dependent gene expression has been well documented $(3,7,26)$. As a result, we wished to determine whether garlic saponins protect cells from intracellular oxidative stress by activating the Nrf 2 signaling pathway. As shown in Fig. 4A and B, treatment of the C2C12 cells with garlic saponins induced Nrf2 expression and the phosphorylation of Nrf2 at Ser40 in a duration- and dose-dependent manner and was associated with the induction of HO-1. However, NQO1 and Keap1 were relatively unaffected by treatment with garlic saponins. We then examined the effect of garlic saponins on the 
A

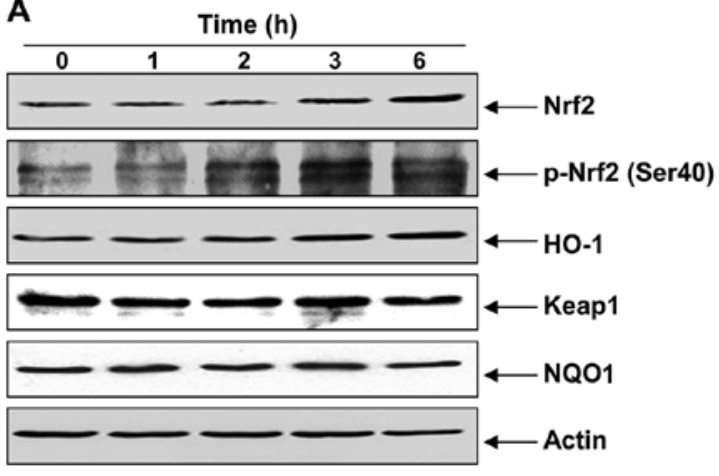

C

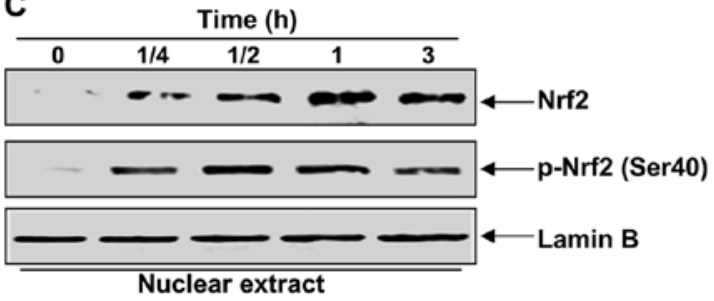

B

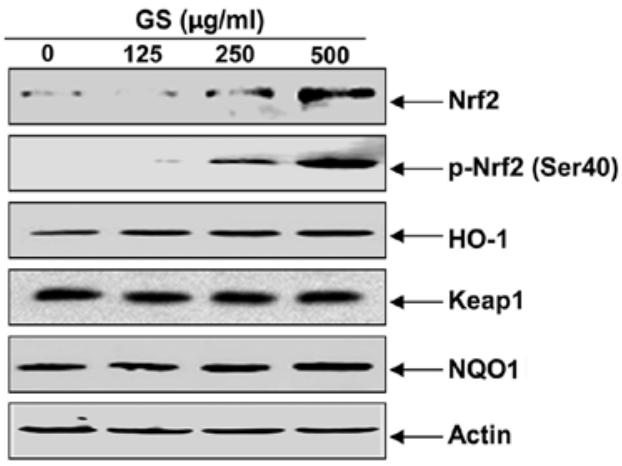

D

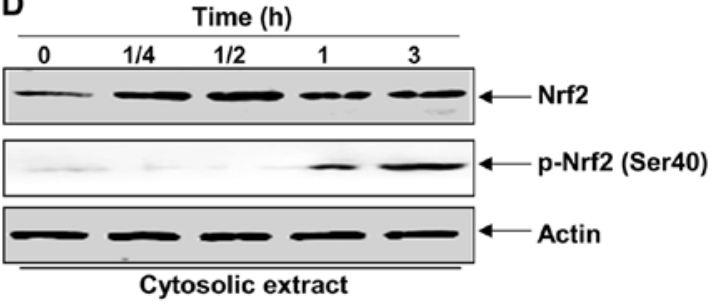

Figure 4. Induction of nuclear factor erythroid 2-related factor 2 (Nrf2) and heme oxygenase-1 (HO-1) expression by garlic saponins in C2C12 cells. Cells were incubated with a $500 \mu \mathrm{g} / \mathrm{ml}$ of garlic saponins for the (A,C and D) specified periods of time or (B) with the specified concentrations of garlic saponins for $6 \mathrm{~h}$. (A and B) Total cellular, (C) nuclear, or (D) cytosolic proteins were separated on SDS-polyacrylamide gels and then transferred onto nitrocellulose membranes. The membranes were probed with the specified antibodies. Proteins were visualized using an ECL detection system. Actin and lamin B were used as the internal controls of total cellular and nuclear proteins, respectively. Keap1; kelch-like ECH-associated protein 1; NQO1, NAD(P)H:quinone oxidoreductase 1; GS, garlic saponins.

A

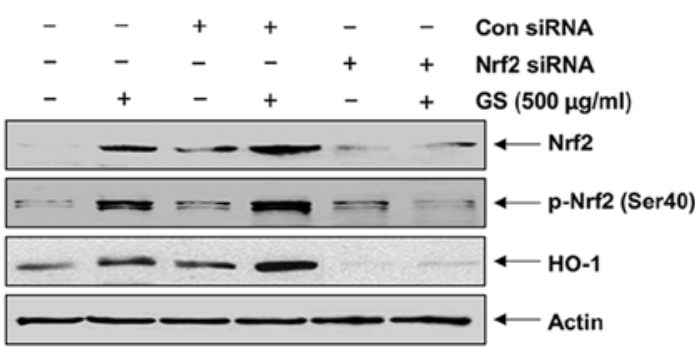

B

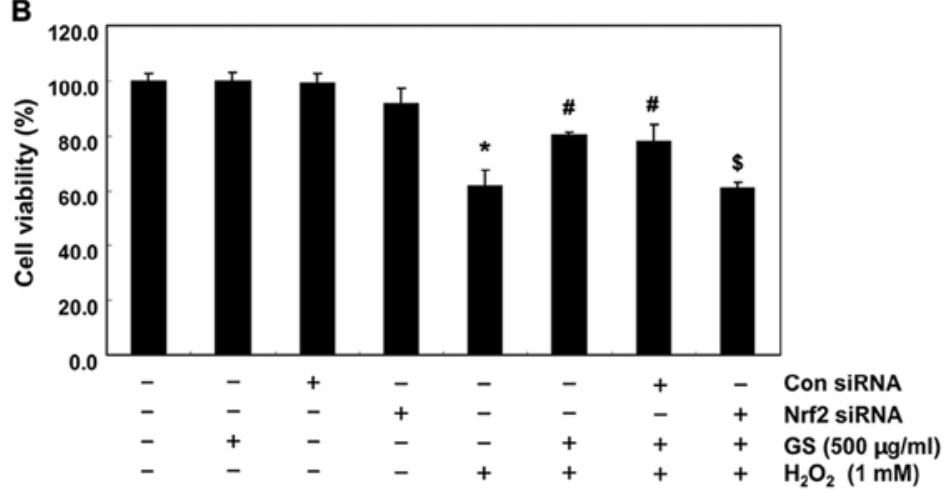

Figure 5. Nuclear factor erythroid 2-related factor 2 (Nrf2)-mediated induction of heme oxygenase-1 (HO-1) expression by garlic saponins in C2C12 cells. Cells were transfected with control (Con siRNA, as negative control for the RNA interference) and Nrf2 siRNA. After $24 \mathrm{~h}$, the cells were treated with a $500 \mu \mathrm{g} / \mathrm{ml}$ of garlic saponins for $6 \mathrm{~h}$ (A) or pre-treated with a $500 \mu \mathrm{g} / \mathrm{ml}$ of garlic saponins for $1 \mathrm{~h}$ and then stimulated with or without $1 \mathrm{mM} \mathrm{H}_{2} \mathrm{O}_{2}$ in the presence of garlic saponins for a further $6 \mathrm{~h}$ (B). (A) Cellular proteins were separated on SDS-polyacrylamide gels and then transferred onto nitrocellulose membranes. The membranes were probed with the specific antibodies against Nrf2, p-Nrf2 and HO-1. Proteins were visualized using an ECL detection system. Actin was used as a loading control (B) Cell viability was estimated by MTT assay. The results are presented as the means \pm SD values obtained from 3 independent experiments ("P $<0.05$ compared with the control group; ${ }^{*} \mathrm{P}<0.05$ compared with the $\mathrm{H}_{2} \mathrm{O}_{2}$-stimulated group; ${ }^{\$} \mathrm{P}<0.05$ compared with the $\mathrm{H}_{2} \mathrm{O}_{2}$ - and garlic saponins-treated group). GS, garlic saponins .

intracellular localization of Nrf2 and found that there was an increased nuclear translocation of phosphorylated Nrf2 proteins following treatment with garlic saponins (Fig. 4C and D).

Garlic saponins upregulate $\mathrm{HO}-1$ expression through the activation of $\mathrm{Nrf} 2$ in $\mathrm{C} 2 \mathrm{C} 12$ cells. We then developed an $\mathrm{Nrf} 2$ gene knockdown model using siRNA transfection to demonstrate the contribution of $\mathrm{Nrf} 2$ signaling to the counteractive effects of garlic saponins on $\mathrm{H}_{2} \mathrm{O}_{2}$-induced cytotoxicity. Western blot analysis revealed that Nrf2 siRNA reduced the expression of Nrf2 and the phosphorylation of Nrf2 induced by treatment with garlic saponins. The expression of HO-1 which was induced by treatment with garlic saponins was also blocked following transfection of the cells with Nrf2 siRNA (Fig. 5A), which is evidence that the augmentation of HO-1 expression is mediated by Nrf2. To confirm the involvement of Nrf2, the protective effects of garlic saponins against the $\mathrm{H}_{2} \mathrm{O}_{2}$-induced reduction in cell viability were determined in cells in which Nrf2 was knocked down. As shown in Fig. 5B, transfection with Nrf2 siRNA cancelled out the cytoprotective effects of garlic saponins when compared with the control siRNA-transfected cells, providing evidence that garlic saponins initiate the cellular antioxidant defense system through the activation of the Nrf2/HO-1 signaling pathway. 


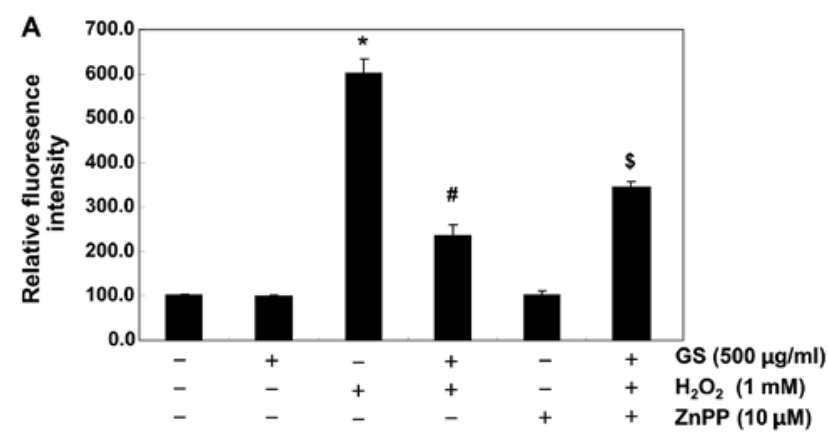

A
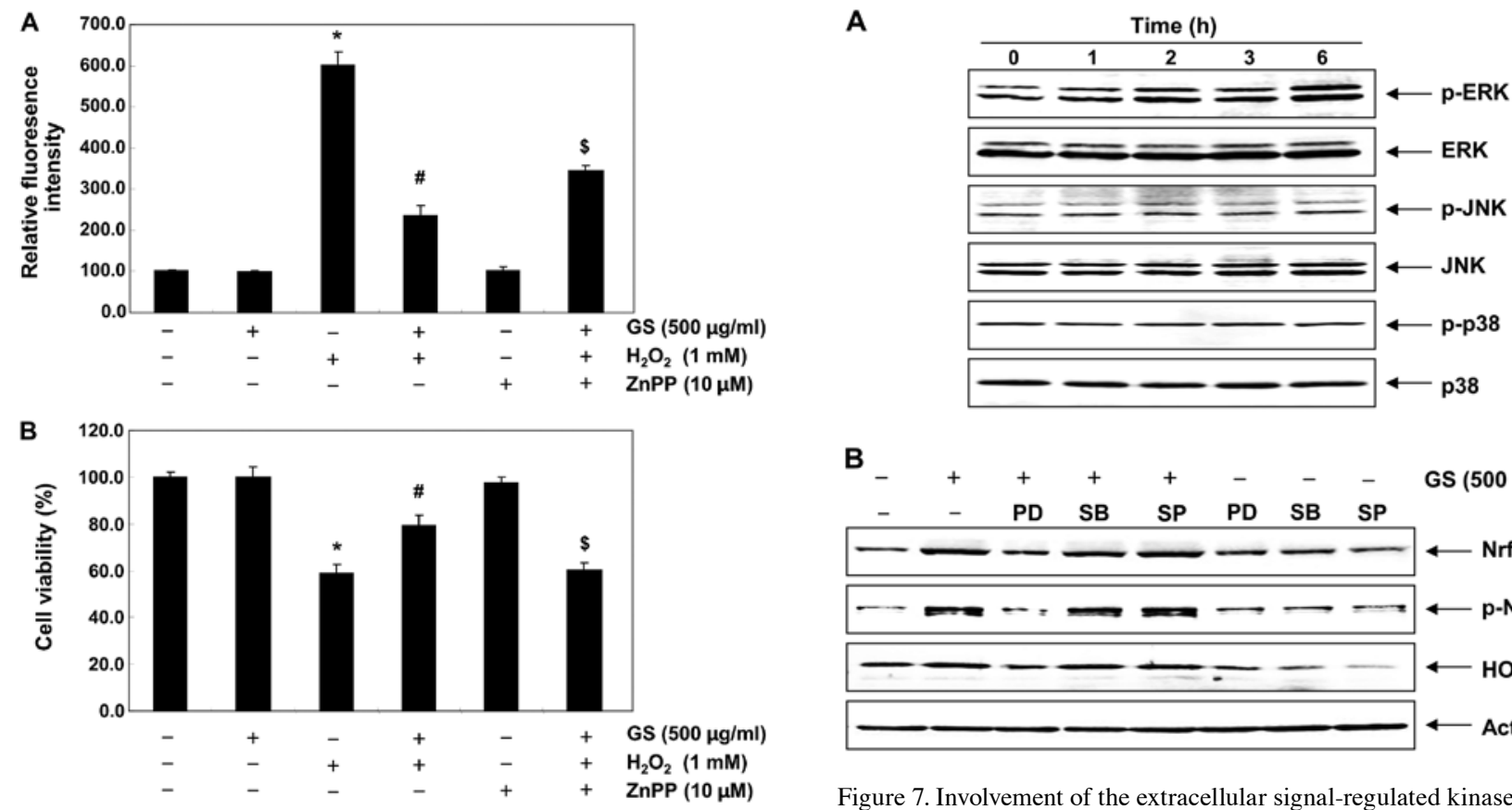

Figure 6. Effects of heme oxygenase-1 (HO-1) inhibitor on the garlic saponinmediated reduction of reactive oxygen species (ROS) formation and growth inhibition by $\mathrm{H}_{2} \mathrm{O}_{2}$ in $\mathrm{C} 2 \mathrm{C} 12$ cells. Cells were pre-treated with $500 \mu \mathrm{g} / \mathrm{ml}$ of garlic saponins for $1 \mathrm{~h}$. The cells were then stimulated with or without $1 \mathrm{mM}$ $\mathrm{H}_{2} \mathrm{O}_{2}$ in the presence of $10 \mu \mathrm{M} \mathrm{ZnPP}$ for a further $6 \mathrm{~h}$. (A) ROS generation and (B) cell viability were then estimated. The results are presented as the means \pm SD values obtained from 3 ndependent experiments $\left({ }^{*} \mathrm{P}<0.05\right.$ compared with the control group; ${ }^{\#} \mathrm{P}<0.05$ compared with the $\mathrm{H}_{2} \mathrm{O}_{2}$-stimulated group; ${ }^{\$} \mathrm{P}<0.05$ compared with the $\mathrm{H}_{2} \mathrm{O}_{2}$ - and garlic saponin-treated group). GS, garlic saponins.

Nrf2/HO-1 pathway is involved in the cytoprotective effects of saponins in $\mathrm{C} 2 \mathrm{Cl} 2$ cells. To provide further confirmation that the antioxidant and cytoprotective activities of garlic saponins against oxidative stress in $\mathrm{C} 2 \mathrm{C} 12$ cells are mediated through the activation of the $\mathrm{Nrf} 2 / \mathrm{HO}-1$ signaling pathway, the $\mathrm{C} 2 \mathrm{C} 12$ cells were pre-incubated with or without $\mathrm{ZnPP}$, a specific inhibitor of HO-1. The ROS levels and cell viability were also assessed. As shown in Fig. 6, ZnPP nullified the protective effect of garlic saponins on the $\mathrm{H}_{2} \mathrm{O}_{2}$-induced production of ROS and the reduction in cell viability. These data suggest that garlic saponins exert their protective effects by activating the cellular defense mechanisms against oxidative stress through the Nrf2related cytoprotective pathway. The subsequent upregulation of HO-1 thus plays a crucial role in the protective effects of saponins in $\mathrm{C} 2 \mathrm{C} 12$ cells.

Garlic saponins induce HO-1 expression through the extracellular signal-regulated kinase (ERK)-Nrf2 signaling pathway. Previous studies have demonstrated that multiple phosphorylation cascades participate in regulating the translocation of Nrf2 and Nrf2-mediated HO-1 gene expression (27-29). To identify the upstream signaling events involved in the activation of Nrf2 and the induction of HO-1 by garlic saponin, the potential involvement of MAPKs was explored. MAPKs are classified into three major subgroups, namely ERK, c-Jun N-terminal kinase (JNK) and p38 MAPK. Although garlic saponins induced the phosphorylation of JNK to a certain extent, it was found that their effect was only significant on the phosphoryla- tion of ERK in a duration-dependent manner. There were no significant changes observed in the levels of phosphorylated p38 MAPK compared with the controls (Fig. 7A). To determine whether garlic saponins induce Nrf2 expression and phosphorylation, and HO-1 expression through the activation of ERK, the cells were pre-treated with garlic saponins for $1 \mathrm{~h}$ and then incubated with MAPK inhibitors. As shown in Fig. 7B, when the cells were incubated with a selective inhibitor of ERK (PD98059), the induction and phosphorylation of Nrf2 were blocked; HO-1 induction was diminished accordingly. However, the p38 MAPK inhibitor (SB203580) and JNK inhibitor (SP600125) were unable to reduce Nrf2 and HO-1 expression and Nrf2 phosphorylation induced by garlic saponins. Taken together, these observations indicate that the way in which garlic saponins activate the Nrf2/HO-1 signaling pathway involves the ERK pathway.

\section{Discussion}

It has been reported that oxidative stress accompanies inflammation, aging, and neurodegenerative and cardiovascular diseases. Oxidative stress can affect the myoblast cytoskeleton and induce cell apoptosis. Both mechanical trauma and prolonged ischemia have been proven to increase the permeability of the plasma membrane for $\mathrm{Ca}^{2+}$, leading to the increased production of ROS $(30,31)$. Chronic inflammation in vivo is also associated with chronic oxidative stress. It has been demonstrated that post-ischemic reperfusion leads to oxidative surges and 
thus has also been cited as a factor in the formation of pressure ulcers $(31,32)$. Although some studies have examined how oxidative stress quantitatively affects the load-carrying capacity of muscle cells $(33,34)$, whether oxidative stress in myoblasts is accompanied by the dysfunction of muscles has not yet been determined. In the present study, as part of the screening program for therapeutic antioxidant agents from traditional food sources, we examined whether garlic saponins offer protection from oxidative stress-induced cytotoxicity using a $\mathrm{C} 2 \mathrm{C} 12$ myoblast cell model. We first observed that, when the $\mathrm{C} 2 \mathrm{C} 12$ myoblasts were treated with garlic saponins in the presence of $\mathrm{H}_{2} \mathrm{O}_{2}$, cell viability recovered significantly due to the inhibition of $\mathrm{H}_{2} \mathrm{O}_{2}$-induced ROS generation, compared to stimulation with $\mathrm{H}_{2} \mathrm{O}_{2}$ alone. Our data also indicated that stimulation with $\mathrm{H}_{2} \mathrm{O}_{2}$ increased the tail length and expression of $\mathrm{p}-\gamma \mathrm{H} 2 \mathrm{AX}$; however, these effects were mitigated in the $\mathrm{C} 2 \mathrm{C} 12$ cells which had been treated with garlic saponins prior to exposure to $\mathrm{H}_{2} \mathrm{O}_{2}$ (Fig. 3). As a result, these findings suggest that garlic saponins are useful for the prevention of $\mathrm{H}_{2} \mathrm{O}_{2}$-induced cytotoxicity due to their prominent antioxidant effects.

It has previoulsy been suggested that the mammalian oxidative stress response is coordinated by the Nrf2 transcription factor. Under normal cellular conditions, $\mathrm{Nrf} 2$ is inactive and bound in the cytosol by Keap1 $(3,4)$. The translocation of Nrf2 into the nucleus is essential for the transactivation of Nrf2-inducible genes, such as those encoding HO-1, which is a key component of protection against oxidative stress $(3,7,26)$. In addition, the phosphorylation of Nrf 2 at Ser40 by several kinases is also a critical process in its stabilization and nuclear translocation (5-7). As illustrated in Fig. 4, we observed that treatment with garlic saponins increased the levels of total and phosphorylated Nrf2, along with the nuclear accumulation of HO-1 (Fig. 5A). In addition, the silencing of Nrf2 halted the protective efects of the garlic saponins on $\mathrm{H}_{2} \mathrm{O}_{2}$-induced growth inhibition of $\mathrm{C} 2 \mathrm{C} 12$ cells (Fig. 5B), and the inhibition of HO-1 function using the HO-1 inhibitor, $\mathrm{ZnPP}$, significantly weakened the protective effects of garlic saponins on $\mathrm{H}_{2} \mathrm{O}_{2}$ induced ROS generation and growth inhibition (Fig. 6). These results suggest that the Nrf2-dependent induction of HO-1 by garlic saponins helps to protect cells against oxidative stress.

A number of studies have suggested that diverse protein kinases are involved in the signals that trigger the Nrf2-Keap1 dissociation, the phosphorylation of $\mathrm{Nrf} 2$ and the antioxidantinduced activation of the Nrf2/HO-1 signaling pathway (8-19). In certain studies, it has been demonstrated that MAPKs play a crucial role in the cellular response to a wide variety of signals elicited by growth factors, hormones and cytokines, and to genotoxic and oxidative stressors $(35,36)$. Recent research has demonstrated that the activation of MAPK signaling leads to the phosphorylation and/or translocation of Nrf2 to the nucleus. For example, the flavonoid, morin, has been shown to upregulate the activity of HO-1 through the ERK/Nrf2 signaling pathway (37). The phenolic glucoside, gastrodin, has also been shown to stimulate HO-1 expression through the activation of the p38 MAPK/Nrf2 signaling pathway (38). In addition, eckol, a phlorotannin isolated from brown algae, has been shown to induce Nrf2-dependent HO-1 expression through the JNK and PI3K/Akt signaling pathways (39). These findings suggest that the role of each pathway in the activation of Nrf2/HO-1 signaling, and their molecular targets, may be specific to the stimulus and cell type. The results of the present study demonstrate that JNK and p38 MAPK are not involved in the activation of Nrf2/HO-1 signaling induced by garlic saponin, since their inhibitors had no effect on garlic saponin-induced HO-1 and Nrf2 expression or Nrf2 phosphorylation. However, the ERK inhibitor, PD98059, suppressed the garlic saponin-induced changes to HO-1 and Nrf2 (Fig. 7B). This suggests that ERK plays a crucial role in the Nrf2-dependent induction of HO-1.

In conclusion, in the present study, we demonstrate that garlic saponins markedly induces Nrf2-mediated HO-1 expression through the ERK/Nrf2 signaling pathway, which contributes, at least in part, to the cellular defense mechanism against oxidative stress-induced genotoxic events. Although such complex molecular mechanisms require further investigation to identify the active saponins contained in crude garlic saponins, the findings of our study suggest that garlic saponins have potential therapeutic value as antioxidant agents.

\section{Acknowledgements}

This study was supported by the R\&D program of MOTIE/KEIT (10040391, Development of Functional Food Materials and Device for Prevention of Aging-associated Muscle Function Decrease) and the National Research Foundation of Korea grant funded by the Korean government (2013 041811, NRF-2014R1A2A1A09006983 and 2015R1A2A2A01004633).

\section{References}

1. Kregel $\mathrm{KC}$ and Zhang $\mathrm{HJ}$ : An integrated view of oxidative stress in aging: basic mechanisms, functional effects, and pathological considerations. Am J Physiol Regul Integr Comp Physiol 292: R18-R36, 2007.

2. Finkel T: Signal transduction by reactive oxygen species. J Cell Biol 194: 7-15, 2011.

3. Venugopal R and Jaiswal AK: Nrf1 and Nrf2 positively and c-Fos and Fra1 negatively regulate the human antioxidant response element-mediated expression of NAD(P)H:quinone oxidoreductasel gene. Proc Natl Acad Sci USA 93: 14960-14965, 1996.

4. Zhang $Y$ and Gordon GB: A strategy for cancer prevention: stimulation of the Nrf2-ARE signaling pathway. Mol Cancer Ther 3: 885-893, 2004.

5. Kaspar JW, Niture SK and Jaiswal AK: Nrf2:INrf2 (Keap1) signaling in oxidative stress. Free Radic Biol Med 47: 1304-1309, 2009.

6. Niture SK, Khatri R and Jaiswal AK: Regulation of Nrf2-an update. Free Radic Biol Med 66: 36-44, 2014.

7. Surh YJ, Kundu JK and Na HK: Nrf2 as a master redox switch in turning on the cellular signaling involved in the induction of cytoprotective genes by some chemopreventive phytochemicals. Planta Med 74: 1526-1539, 2008.

8. Qaisiya M, Coda Zabetta CD, Bellarosa C and Tiribelli C: Bilirubin mediated oxidative stress involves antioxidant response activation via Nrf2 pathway. Cell Signal 26: 512-520, 2014.

9. Nguyen CN, Kim HE and Lee SG: Caffeoylserotonin protects human keratinocyte $\mathrm{HaCaT}$ cells against $\mathrm{H}_{2} \mathrm{O}_{2}$-induced oxidative stress and apoptosis through upregulation of HO-1 expression via activation of the PI3K/Akt/Nrf2 pathway. Phytother Res 27 : 1810-1818, 2013.

10. Bates DJ, Smitherman PK, Townsend AJ, King SB and Morrow CS: Nitroalkene fatty acids mediate activation of Nrf2/ARE-dependent and PPAR $\gamma$-dependent transcription by distinct signaling pathways and with significantly different potencies. Biochemistry 50: 7765-7773, 2011.

11. Landete JM: Dietary intake of natural antioxidants: vitamins and polyphenols. Crit Rev Food Sci Nutr 53: 706-721, 2013.

12. Kwak JS, Kim JY, Paek JE, Lee YJ, Kim HR, Park DS and Kwon O: Garlic powder intake and cardiovascular risk factors: a meta-analysis of randomized controlled clinical trials. Nutr Res Pract 8: 644-654, 2014. 
13. Rana SV, Pal R, Vaiphei K, Sharma SK and Ola RP: Garlic in health and disease. Nutr Res Rev 24: 60-71, 2011.

14. Capasso A: Antioxidant action and therapeutic efficacy of Allium sativum L. Molecules 18: 690-700, 2013.

15. Nencini C, Menchiari A, Franchi GG and Micheli L: In vitro antioxidant activity of aged extracts of some Italian Allium species. Plant Foods Hum Nutr 66: 11-16, 2011.

16. Lanzotti V, Barile E, Antignani V, Bonanomi G and Scala F: Antifungal saponins from bulbs of garlic, Allium sativum L. var. Voghiera. Phytochemistry 78: 126-134, 2012.

17. Khalil WK, Ahmed KA, Park MH, Kim YT, Park HH and Abdel-Wahhab MA: The inhibitory effects of garlic and Panax ginseng extract standardized with ginsenoside $\operatorname{Rg} 3$ on the genotoxicity, biochemical, and histological changes induced by ethylenediaminetetraacetic acid in male rats. Arch Toxicol 82: 183-195, 2008

18. Amagase H: Clarifying the real bioactive constituents of garlic J Nutr 136 (Suppl 3): 716S-725S, 2006.

19. Matsuura H: Saponins in garlic as modifiers of the risk of cardiovascular disease. J Nutr 131 (3s): 1000S-1005S, 2001.

20. Lacaille-Dubois MA and Wagner H: A review of the biological and pharmacological activities of saponins. Phytomedicine 2 : 363-386, 1996.

21. Fehresti Sani M, Montasser Kouhsari S and Moradabadi L: Effects of three medicinal plants extracts in experimental diabetes: antioxidant enzymes activities and plasma lipids profiles in comparison with metformin. Iran J Pharm Res 11: 897-903, 2012.

22. Luo H, Huang J, Liao WG, Huang QY and Gao YQ: The antioxidant effects of garlic saponins protect $\mathrm{PC} 12$ cells from hypoxia-induced damage. Br J Nutr 105: 1164-1172, 2011.

23. Song JL, Choi JH, Seo JH, Kil JH and Park KY: Antioxidative effects of fermented sesame sauce against hydrogen peroxide-induced oxidative damage in LLC-PK1 porcine renal tubule cells. Nutr Res Pract 8: 138-145, 2014

24. Kang JS, Han MH, Kim GY, Kim CM, Kim BW, Hwang HJ and Hyun Y: Nrf2-mediated HO-1 induction contributes to antioxidant capacity of a Schisandrae Fructus ethanol extract in C2C12 myoblasts. Nutrients 6: 5667-5678, 2014.

25. Rogakou EP, Pilch DR, Orr AH, Ivanova VS and Bonner WM: DNA double-stranded breaks induce histone $\mathrm{H} 2 \mathrm{AX}$ phosphorylation on serine 139. J Biol Chem 273: 5858-5868, 1998.

26. Kim SJ, Ho Hur J, Park C, Kim HJ, Oh GS, Lee JN, Yoo SJ, Choe SK, So HS, Lim DJ, Moon SK and Park R: Bucillamine prevents cisplatin-induced ototoxicity through induction of glutathione and antioxidant genes. Exp Mol Med 47: e142, 2015.
27. Pischke SE, Zhou Z, Song R, Ning W, Alam J, Ryter SW and Choi AM: Phosphatidylinositol 3-kinase/Akt pathway mediates heme oxygenase-1 regulation by lipopolysaccharide. Cell Mol Biol (Noisy-le-grand) 51: 461-470, 2005.

28. Paine A, Eiz-Vesper B, Blasczyk R and Immenschuh S: Signaling to heme oxygenase- 1 and its anti-inflammatory therapeutic potential. Biochem Pharmacol 80: 1895-1903, 2010.

29. Yang JJ, Tao H, Huang C and Li J: Nuclear erythroid 2-related factor 2: a novel potential therapeutic target for liver fibrosis. Food Chem Toxicol 59: 421-427, 2013.

30. Abruzzo PM, Esposito F, Marchionni C, di Tullio S, Belia S, Fulle S, Veicsteinas A and Marini M: Moderate exercise training induces ROS-related adaptations to skeletal muscles. Int J Sports Med 34: 676-687, 2013.

31. Kumar S, Kain V and Sitasawad SL: High glucose-induced $\mathrm{Ca}^{2+}$ overload and oxidative stress contribute to apoptosis of cardiac cells through mitochondrial dependent and independent pathways. Biochim Biophys Acta 1820: 907-920, 2012.

32. Li NS, Luo XJ,Zhang YS, He L, Liu YZ and Peng J: Phloroglucinol protects gastric mucosa against ethanol-induced injury through regulating myeloperoxidase and catalase activities. Fundam Clin Pharmacol 25: 462-468, 2011.

33. Klimathianaki M, Vaporidi K and Georgopoulos D: Respiratory muscle dysfunction in COPD: from muscles to cell. Curr Drug Targets 12: 478-488, 2011.

34. Yao Y, Xiao Z, Wong S, Hsu YC, Cheng T, Chang CC, Bian L and Mak AF: The effects of oxidative stress on the compressive damage thresholds of $\mathrm{C} 2 \mathrm{C} 12$ mouse myoblasts: implications for deep tissue injury. Ann Biomed Eng 43: 287-296, 2015.

35. Kim EK and Choi EJ: Pathological roles of MAPK signaling pathways in human diseases. Biochim Biophys Acta 1802: 396-405, 2010.

36. Winter-Vann AM and Johnson GL: Integrated activation of MAP3Ks balances cell fate in response to stress. J Cell Biochem 102: 848-858, 2007.

37. Park JY, Kang KA, Kim KC, Cha JW, Kim EH and Hyun JW: Morin induces heme oxygenase-1 via ERK-Nrf2 signaling pathway. J Cancer Prev 18: 249-256, 2013.

38. Jiang G, Hu Y, Liu L, Cai J, Peng C and Li Q: Gastrodin protects against MPP(+)-induced oxidative stress by up regulates heme oxygenase-1 expression through p38 MAPK/Nrf2 pathway in human dopaminergic cells. Neurochem Int 75: 79-88, 2014.

39. Jun YJ, Lee M, Shin T, Yoon N, Kim JH and Kim HR: eckol enhances heme oxygenase-1 expression through activation of Nrf2/JNK pathway in HepG2 cells. Molecules 19: 15638-15652, 2014. 